\title{
Erratum to: "Like a lots happened with my whole childhood": violence, trauma, and addiction in pregnant and postpartum women from Vancouver's Downtown Eastside
}

Iris Torchalla ${ }^{1,2^{*}}$, Isabelle Aube Linden ${ }^{1}$, Verena Strehlau ${ }^{1,2}$, Erika K. Neilson ${ }^{1}$ and Michael Krausz ${ }^{1,2,3}$

\section{Erratum}

This article [1] was unintentionally published twice in this journal, by the same authors. The following should be considered the version of record and used for citation purposes: "Torchalla et al.: "Like a lots happened with my whole childhood": violence, trauma, and addiction in pregnant and postpartum women from Vancouver's Downtown Eastside, Harm Reduction Journal (2014) 11:34, https://doi.org/10.1186/1477-7517-11-34". The duplicate article [2] is to be ignored. We apologize for not detecting the duplication during the publication process.

\footnotetext{
Author details

${ }^{1}$ Centre for Health Evaluation and Outcome Sciences (CHÉOS), St. Paul's Hospital, 588-1081 Burrard Street, Vancouver, BC V6Z 1Y6, Canada. ${ }^{2}$ Department of Psychiatry, University of British Columbia, Detwiller Pavilion, 2255 Westbrook Mall, Vancouver, BC V6T 2A1, Canada. ${ }^{3}$ School of Population and Public Health, University of British Columbia, James Mather Building; 5804 Fairview Avenue, Vancouver, BC V6T 1Z3, Canada.
}

Received: 6 September 2017 Accepted: 15 September 2017 Published online: 19 September 2017

\section{References}

1. Torchalla, et al. "Like a lots happened with my whole childhood": violence, trauma, and addiction in pregnant and postpartum women from Vancouver's Downtown Eastside. Harm Reduct. J. 2014;11:34. doi:10.1186/1477-7517-11-34.

2. Torchalla, et al. "Like a lots happened with my whole childhood": violence, trauma, and addiction in pregnant and postpartum women from Vancouver's Downtown Eastside. Harm Reduct. J. 2015;12:1. doi:10.1186/1477-7517-12-1.

\footnotetext{
* Correspondence: itorchalla@cheos.ubc.ca

${ }^{1}$ Centre for Health Evaluation and Outcome Sciences (CHÉOS), St. Paul's Hospital, 588-1081 Burrard Street, Vancouver, BC V6Z 1Y6, Canada ${ }^{2}$ Department of Psychiatry, University of British Columbia, Detwiller Pavilion, 2255 Westbrook Mall, Vancouver, BC V6T 2A1, Canada
} 